Bulletin of Mathematical Biology manuscript No.

(will be inserted by the editor)

\title{
Evolutionarily stable strategies for fecundity and swimming speed of fish
}

\author{
Michael J. Plank • Jonathan W. \\ Pitchford · Alex James
}

the date of receipt and acceptance should be inserted later

\begin{abstract}
Many pelagic fish species have a life history that involves producing a large number of small eggs. This is the result of a trade-off between fecundity and larval survival probability. There are also trade-offs involving other traits, such as larval swimming speed. Swimming faster increases the average food encounter rate but also increases the metabolic cost. Here we introduce an evolutionary model comprising fecundity and swimming speed as heritable traits. We show that there can be two evolutionary stable strategies. In environments where there is little noise in the food encounter rate, the stable strategy is a low-fecundity strategy with a swimming speed that minimises the mean time taken to reach reproductive maturity. However, in noisy environments, for example where the prey distribution is patchy or the water is turbulent, strategies that optimise mean outcomes are often outperformed by strategies that increase inter-individual variance. We show that, when larval growth rates are unpredictable, a high-fecundity strategy is evolutionarily stable. In a population following this strategy, the swimming speed is higher than would be anticipated by maximising the mean growth rate.
\end{abstract}

Keywords first passage time $\cdot$ fish egg size $\cdot$ fish growth rate $\cdot$ genetic algorithm $\cdot$ patchiness $\cdot$ stochastic growth

M. J. Plank

School of Mathematics and Statistics, University of Canterbury, Christchurch 8140, New Zealand and Te Pūnaha Matatini, a New Zealand Centre of Research Excellence. E-mail: michael.plank@canterbury.ac.nz

J. W. Pitchford

York Centre for Complex Systems Analysis, University of York, United Kingdom. E-mail: jon.pitchford@york.ac.uk

A. James

School of Mathematics and Statistics, University of Canterbury, Christchurch 8140, New Zealand and Te Pūnaha Matatini, a New Zealand Centre of Research Excellence. E-mail: alex.james@canterbury.ac.nz 


\section{Introduction}

Newborn larvae of pelagic spawning fish species face an extremely uncertain outlook. The median egg diameter of marine fish species is approximately 1.1 mm (Chambers and Leggett, 1996). These tiny eggs are abandoned by their parents in turbulent water (MacKenzie and Kiørboe, 1995), with a highly patchy prey distribution (Tsuda et al., 1993), spatiotemporal variations in abiotic factors like temperature and salinity (Jennings and Warr, 2003) and an abundance of predators. Most quickly perish - larval mortality rates as high as $42 \%$ per day have been recorded in Atlantic mackerel (Ware and Lambert, 1985) - but a lucky few reach reproductive maturity and contribute to the generation of spawners.

Most marine fish species have very high fecundity (Elgar, 1990). This high fecundity, high mortality life history skews the evolutionary pressures that operate on these populations because only the extreme tail of the fitness distribution contributes to the next generation. For this reason, the inter-individual variance in metrics such as the prey encounter rate can be a more important determinant of reproductive fitness than the mean (Pitchford et al., 2005).

Larval swimming speed is an important factor affecting the expected prey encounter rate (Chick and Van Den Avyle, 2000). There are trade-offs involving this trait: swimming faster will increase the prey encounter rate but will also increase energy expenditure. Previous models of swimming speed (Darowski et al., 1988; Pitchford et al., 2003) have focused on maximising the mean net rate of energy gain or mean growth rate. However, because only a tiny fraction of larvae reach reproductive maturity, the important part of the distribution of growth rates is the tail and focusing on optimising mean values may not produce representative results (Pitchford et al., 2005). In such circumstances, a strategy with a lower mean growth rate but a higher variance in the growth rate may be evolutionarily advantageous. This can occur if some fitness effects of a heritable trait are subject to higher demographic stochasticity (inter-individual variance) than others (Currey et al., 2007). Increasing swimming speed has an energetic cost that is approximately deterministic because it is a function of the energy required to overcome the drag force of the water (Pitchford et al., 2003). In contrast, the energetic benefit of swimming faster is stochastic because prey distributions are often very patchy (Duarte and Alcaraz, 1989).

The trade-offs between fecundity and egg size have been studied empirically and theoretically (Elgar, 1990; Winemiller and Rose, 1993; Andersen et al., 2008). Pelagic-spawning marine species tend to have very high fecundity and very small egg sizes, whereas demersal spawners and freshwater species typically produce fewer, larger eggs, possibly due to lower inter-larval variability (Duarte and Alcaraz, 1989). Models with a predetermined mortality rate cannot fully capture the trade-offs between high and low fecundity strategies because the mortality rate is a major factor influencing optimal life histories. We develop a model to investigate the evolutionarily stable strategy (ESS) for fish that captures trade-offs between fecundity, mortality, prey encounter rate 
and metabolic cost in a stochastic environment. The model includes swimming speed and fecundity as heritable traits. We explore evolutionarily stable strategies for this combination of traits using two methods: (i) maximising an an analytical expression for reproductive fitness; (ii) simulating a genetic algorithm. We show that high fecundity tends to be stable and that, in a noisy environment, high swimming speeds can increase fitness despite reducing the expected net growth rate.

\section{Model}

Larval fish grow as a result of encountering suitable prey and also have a metabolic cost that increases with energy used swimming. We model the body mass $X(t)$ of an individual fish by a stochastic differential equation (SDE):

$$
d X=r d t+\sigma d W, \quad X(0)=x_{0}
$$

(Pitchford et al., 2005), where $W(t)$ is a standard Brownian motion. This is a drift-diffusion process for body size with fixed mean growth rate $r$ and diffusivity $\sigma^{2}$. We assume that reproductive maturity is defined by reaching a certain minimum body size $x_{m}$.

Pitchford and Brindley (2001) modelled foraging in a patchy environment using Poisson processes for patch encounters and for prey encounters within a patch. They calculated the mean and variance of the number of prey encounters per unit time as functions of the average prey density, the patchiness and the predator swimming speed. The mean number of prey encounters $N_{p}$ during a period of time $\delta t$ is independent of the patchiness and is an approximately linear function of the swimming speed $v$ :

$$
E\left(N_{p}\right)=(a+b v) \delta t,
$$

where $a$ and $b$ are constants representing respectively the contribution of turbulence to the encounter rate and the average prey density. Pitchford and Brindley (2001) also showed that the variance in the number of prey encounters increases with the patchiness of the prey distribution. We therefore set

$$
\operatorname{Var}\left(N_{p}\right)=S E\left(N_{p}\right)=S(a+b v) \delta t,
$$

where the constant $S$ represents patchiness. For a homogeneous prey distribution, the number of encounters $N_{p}$ is a Poisson random variable, which implies that $\operatorname{Var}\left(N_{p}\right)=E\left(N_{p}\right)$ and hence $S=1$. Increasingly patchy distributions of the same mean density (e.g. sparse but highly densely populated patches of prey) are represented by values of $S$ greater than 1 (Pitchford and Brindley, 2001). Values of $S$ less than 1 could also occur if there was a regular distribution of prey or a minimum handling time between successive encounters. All prey encounters are assumed to result in an increase in predator body mass of an equal amount $x_{p}$. 
The larval fish experience an metabolic cost due to swimming. This is assumed be deterministic and proportional to the Stokes drag, which increases quadratically with swimming speed (Pitchford and Brindley, 2001). The metabolic cost is assumed to result in a loss of body mass at rate

$$
E_{\text {swim }}=c v^{2} .
$$

According to the above assumptions, the mean net growth rate and the diffusivity in Eq. (2.1) are

$$
\begin{aligned}
r(v) & =(a+b v) x_{p}-c v^{2}, \\
\sigma^{2}(v) & =S(a+b v) x_{p}^{2} .
\end{aligned}
$$

By a non-dimensionalisation of the variables $X, t$ and $v$ (see Appendix), we may rewrite

$$
\begin{aligned}
r(v) & =\alpha+2 v-v^{2}, \\
\sigma^{2}(v) & =s(\alpha+2 v),
\end{aligned}
$$

where $s=S x_{p} / x_{m}, \alpha=4 a c /\left(b^{2} x_{p}\right)$, the initial mass is $X(0)=x_{0} / x_{m}$ and the maturity size is $x_{m}=1$.

The time taken to reach maturity $T_{h}$ is the first time that the body size $X(t)$ exceeds $x_{m}=1$. Since the growth process is stochastic, $T_{h}$ is a random variable, referred to as a first hitting time. For the stochastic process described by Eq. (2.1), the first hitting time has as an inverse Gaussian distribution, with cumulative density function $(\mathrm{CDF})$

$P\left(T_{h}<t\right)=C\left(t ; v, x_{0}\right)=1-\Phi\left(\frac{1-x_{0}-r t}{\sigma \sqrt{t}}\right)+e^{2 r\left(1-x_{0}\right) / \sigma^{2}} \Phi\left(\frac{-1+x_{0}-r t}{\sigma \sqrt{t}}\right)$

where $\Phi$ is the CDF of the standard normal distribution $N(0,1)$ (Grimmett and Stirzaker, 1992). Eq. (2.4) corresponds to the probability that a fish with swimming speed $v$ and mass-at-birth $x_{0}$ will reach maturity by time $t$.

We assume that, on reaching maturity, a constant proportion $p$ of the parent's body mass is used to generate offspring, and that this mass is divided evenly between all $n$ offspring. Hence, the mass-at-birth of an individual whose parent has fecundity $n$ is $x_{0}=p / n$.

The model therefore has three parameters: (i) the mean food intake rate for a non-swimming larvae $\alpha$; (ii) the proportion $p$ of parental mass used for reproduction; and (iii) the noise level $s$, which is the product of the prey patchiness $S$ and the mass of a prey item $x_{p}$ relative to the maturity mass $x_{m}$. We will set $a=0$ (and as a consequence $\alpha=0$ ) so that the expected encounter rate for a non-swimming fish larvae is zero, and $p=0.2$, and investigate a range of noise levels $s$. Note that setting $\alpha=0$ does not remove noise from the system; turbulence and spatial heterogeneity still play an important role in promoting variability in realised encounter rates via the noise parameter $s$. We have tested the effect of varying $\alpha$ in the range 0 to 4 and $p$ in the range 0.05 to 0.5 and this does not qualitatively change the results. Values for $x_{p} / x_{m}$ could range from $10^{-8}$ for the larvae of large species foraging on small prey such as copepods, to $10^{-4}$ for smaller species feeding on larger prey. 


\subsection{Fitness}

In an equilibrium population in which all individuals have fecundity $n$, an average proportion $1 / n$ of all offspring reach reproductive maturity. The assumption of an equilibrium population size is reasonable over evolutionary time scales. For example, this might represent a population whose sustainable size is limited to some carrying capacity by the availability of suitable habitat or the abundance of prey.

The fitness $W(v, n)$ of a strategy with swimming speed $v$ and fecundity $n$ is defined by the expected time taken for a proportion $1 / n$ of the offspring to reach maturity. Since the initial mass of offspring of a parent with fecundity $n$ is $x_{0}=p / n$, the expected time taken, $t^{*}(v, n)$, for a proportion $1 / n$ of the parent's offspring to reach maturity is defined implicitly by

$$
C\left(t^{*} ; v, p / n\right)=1 / n \text {. }
$$

For given values of $v$ and $n$, this equation was solved to find $t^{*}$ using Matlab's numerical root finder fzero. We define fitness $W$ as inversely proportional to $t^{*}:$

$$
W(v, n)=1 / t^{*}(v, n) .
$$

A resident strategy $\left(v_{r}, n_{r}\right)$ is vulnerable to invasion by any mutant strategy $(v, n)$ with a higher fitness. In this model formulation, the fitness of a given strategy is independent of the strategies being followed by other individuals in the population. An ESS is a strategy that cannot be invaded by any mutant strategy (Maynard-Smith, 1982). In this context, an ESS is simply a strategy with higher fitness than any other strategy.

\subsection{Genetic algorithm}

We simulated the evolution of swimming speed and fecundity using a simple genetic algorithm (GA). We assume an adult population of fixed size $N$ and with swimming speed and fecundity traits $\left(v_{i}, n_{i}\right), i=1, \ldots, N$. For each parent $i$, we create $n_{i}$ offspring with traits $\left(v_{i}+\phi_{j}, n_{i} e^{\psi_{j}}\right)$, where $\phi_{j}$ and $\psi_{j}$ $\left(j=1, \ldots, n_{i}\right)$ are independent normal random variables with mean zero and variance $\sigma_{v}^{2}$ and $\sigma_{n}^{2}$ respectively. This represents normally distributed mutations in swimming speed $v$ and in $\log$-fecundity $\ln (n)$. Both traits are restricted to pre-defined ranges $v_{i} \geq 0$ and $1 \leq n_{i} \leq n_{\max }$. If any trait values are generated outside the allowed range, they are adjusted to the closest allowable value. The upper limit on fecundity is interpreted as a physiological limitation on the minimum size for viable offspring $\left(p / n_{\max }\right)$; the effect of varying the parameter $n_{\max }$ will be investigated. The limitation of traits to defined ranges is necessary numerically. The results show that this is not important in terms of swimming speed (intermediate speeds are evolutionarily selected). However, the evolutionary optimum for fecundity commonly lies at one of the physiologically imposed limits, favouring either a strategy of "as many small larvae as possible", or its opposite, depending on the selective environment. 

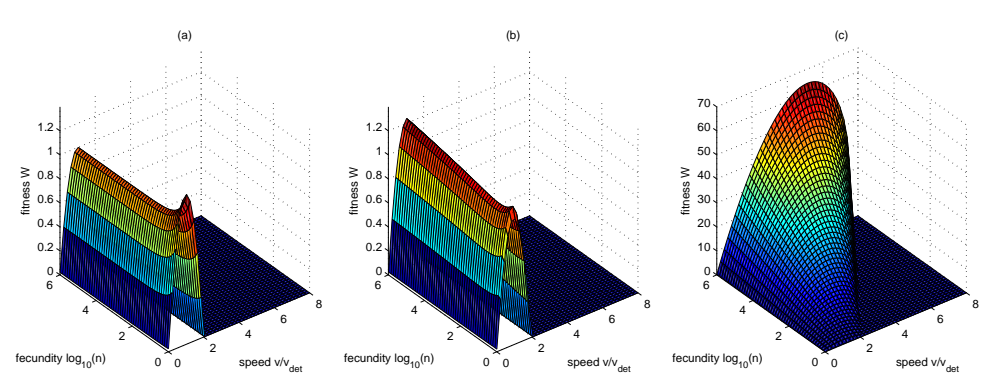

Fig. 1 Fitness calculated using Eq. (2.5) against speed and fecundity. (a) No noise $(s=0)$, highest fitness occurs at fecundity $n=1$ and the deterministically optimum speed $v=v_{\text {det }}$. (b) Moderate noise $(s=0.001)$, highest fitness occurs at maximum fecundity $n=n_{\max }=$ $10^{6}$ and the deterministically optimum swimming speed. (c) High noise $(s=0.4)$, highest fitness occurs at maximum fecundity $n=n_{\max }$ and high swimming speed $v>v_{\text {det }}$.

The total number of offspring is $\sum_{i=1}^{N} n_{i}$. We sample the hitting times of all offspring independently from the distribution in Eq. (2.4) and select the $N$ individuals with the lowest hitting times to make up the next generation of adults. Within these $N$ individuals, we ignore any advantage to reaching maturity earlier and assume that consecutive generations are non-overlapping. This is the simplest selection choice for the GA. An ecological interpretation would be a situation where there is a finite resource or limited space, to which only the most successful $N$ individuals have equal access. More complex selection criteria could be included in the GA, based, for example, on a weighting of the hitting time. However, this would be at the expense of introducing further parameters, which would make the model more difficult to interpret.

\section{Results}

For fixed swimming speed $v$, fitness is a convex function of fecundity. Therefore, the optimal fecundity is always either the minimum allowed fecundity (1) or the maximum allowed fecundity $\left(n_{\max }\right)$. Which of these two strategies has the higher fitness depends on the level of noise. In a low noise $(s \ll 1)$ environment, the low fecundity strategy of having one large offspring is optimal. The optimal swimming speed under these conditions is the one that maximises the mean net growth rate in Eq. (2.2) (i.e. the deterministically optimum speed $\left.v_{\operatorname{det}}=1\right)$. However, as the amount of noise increases, there is a tipping point and, above that point, the optimal strategy is to have as many offspring as physiologically possible. This can be understood intuitively as follows. In an environment where everyone produces a large number of offspring, the larvae all begin life at a very small size. Increasing the number of offspring causes a negligible reduction in the initial larval size and, therefore, negligible change in the hitting time distribution. So having more offspring is like getting more lottery tickets for the same total cost. 
Close to the tipping point between the low fecundity and high fecundity strategies, the optimal swimming speed is still close to the deterministic optimum. However, as the noise level $s$ increases further, the optimal swimming speed increases above the deterministic optimum. This reduces the mean growth rate but, because the metabolic swimming cost is deterministic, it increases the variance of the growth rate. For high levels of noise, the optimal swimming speed can be several times larger than the deterministic optimum. In these cases, the mean growth rate is actually negative (i.e. mass lost due to the metabolic cost of swimming is greater than the mass gained from prey encounters) but the increase in variance means that the individuals in the tail of the hitting-time distribution reach maturity more rapidly and therefore have higher fitness.

Each row of graphs in Fig. 2 shows a single simulation of the GA. The simulations shown are representative of the behaviour of the GA: there is variability among realisations in the time taken to converge, but the longterm behaviour is always the same for a given noise level $s$ and given initial conditions. The results of the GA are consistent with the fitness functions in Fig. 1. The population converges to low fecundity $(n=1)$ when noise is low (Fig. 2a, b) and to high fecundity $\left(n=n_{\max }\right)$ when noise is high (Fig. 2eh). This finding is robust to changes to the value of $n_{\max }$, which only affect the time taken for the population to converge. At moderate noise levels, the population can get trapped in the local optimum at low fecundity (Fig. 2c, d). Whenever there is any noise $(s>0)$, the low fecundity optimum (if it exists) is always local in the sense that fitness is eventually an increasing function of fecundity when fecundity is sufficiently high. The only thing that can make the low-fecundity strategy globally optimal is the physiological restriction on the maximum number of eggs.

Figure 3 shows the optimal trait values according to the analytical model together with the mean trait values after 500 generations of the GA. The GA was run for a range of noise levels $s$ and, for each new noise level, was initialised with the resulting population (i.e. same set of trait values) from the previous noise level. The noise level $s$ was first increased in logarithmic increments from $10^{-8}$ to 1 and then decreased back down to $10^{-8}$. This allows us to investigate bistability in the fecundity, which is indicated by the two branches of results in Fig. 3a. In the range of noise levels for which there are two branches, the population can evolve towards either the low fecundity $(n \approx 1)$ or high fecundity ( $n \approx n_{\max }$ ) strategy, depending on the initial conditions.

The optimal swimming speed is largely independent of initial conditions and consistent with the predictions of the analytical model (Fig. 3b). At low noise levels, when the population is in the low-fecundity state, the swimming speed is close to the value that maximises the mean net growth rate in Eq. (2.2), referred to as the deterministically optimum speed. There is a range of intermediate noise levels (roughly $10^{-4}<s<10^{-2}$ ) for which the optimum strategy is high fecundity but the swimming speed is still close to the deterministic optimum. At high noise levels $\left(s>10^{-2}\right)$, the optimum strategy is high fecundity and high swimming speed. As the noise level is subsequently 

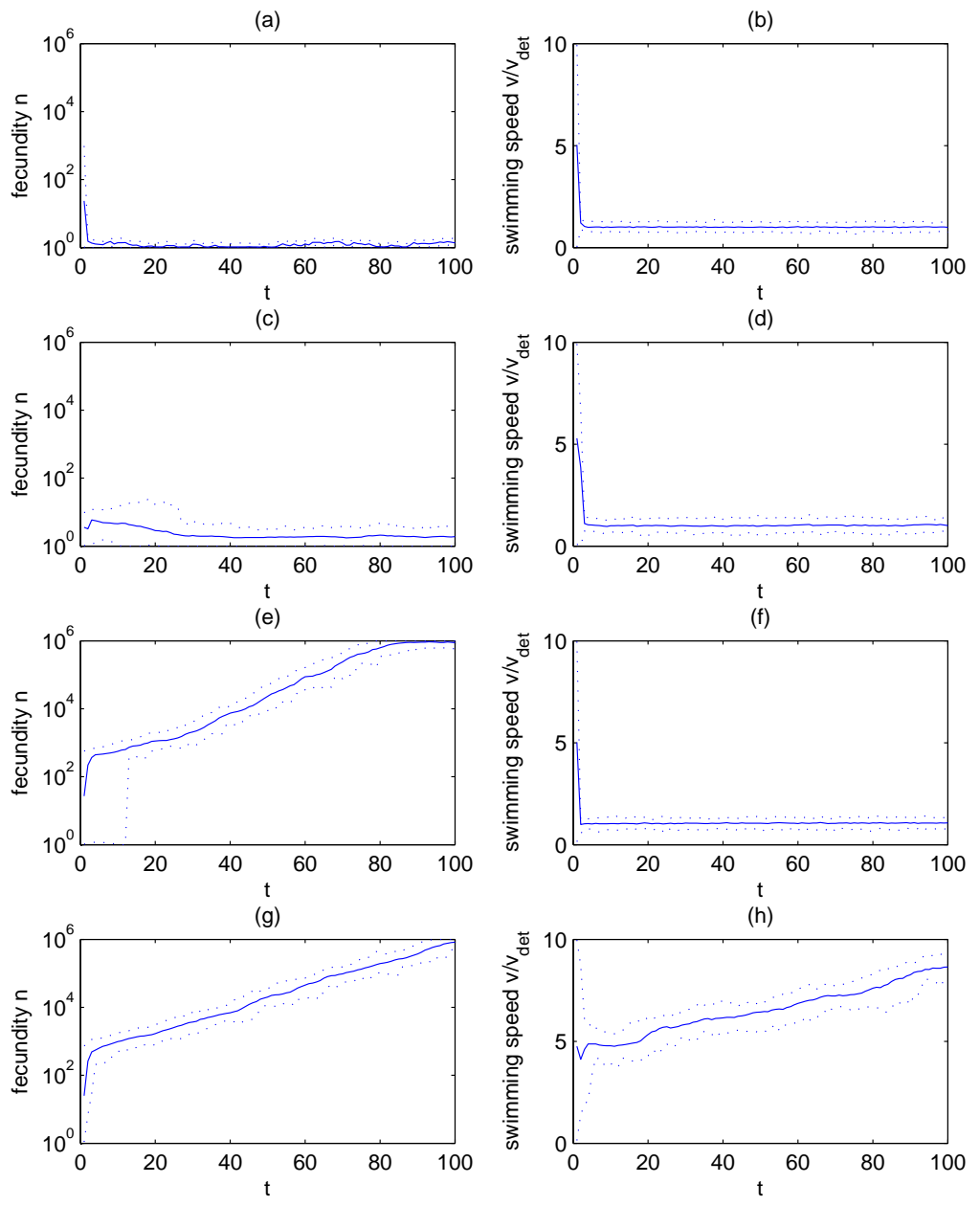

Fig. 2 GA results showing the minimum, mean and maximum trait values of a population of $N=100$ individuals over time ( $t$ represents generation number). (a, b) No noise $(s=0)$, population converges to fecundity $n=1$ and swimming speed $v=v_{\mathrm{det}}$. (c, d, e, f) Moderate noise $(s=0.001)$, population can converge to the local optimum at low fecundity (c), or to the global optimum at high fecundity (e), depending on initial conditions. ( $\mathrm{g}, \mathrm{h}$ ) High noise $(s=1)$, population always converges to the high fecundity optimum with $v>$ $v_{\text {det }}$. Population size $N=100$; mutation size constants $\sigma_{n}=0.1, \sigma_{v}=0.1 v_{\text {det }}$; maximum fecundity $n_{\max }=10^{6}$. Initial swimming speeds uniformly distributed in $\left[0,10 v_{\text {det }}\right]$. In (a, $\mathrm{b}, \mathrm{e}-\mathrm{h})$ initial fecundity is log-uniformly distributed in $[0,1000]$; in $(\mathrm{c}, \mathrm{d})$ initial fecundity is log-uniformly distributed in $[0,10]$. 

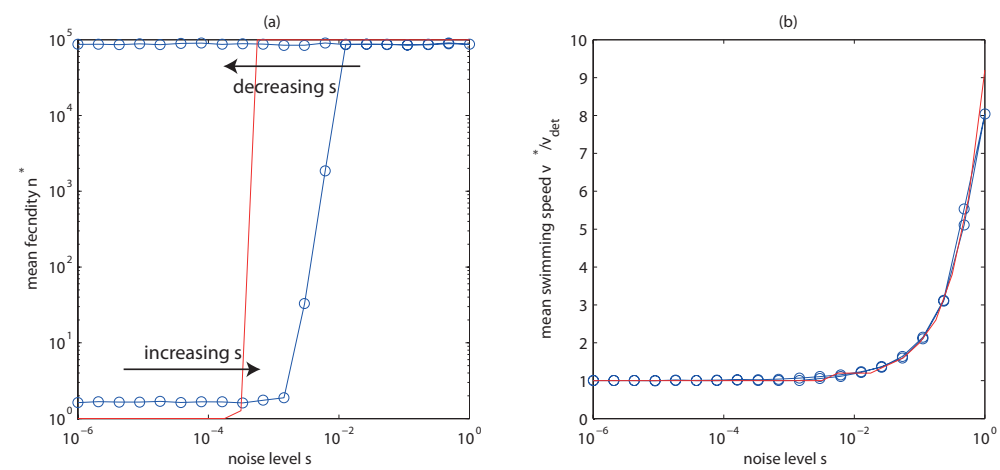

Fig. 3 Fecundity $n$ and swimming speed $v$ against noise level $s$. Blue curves show the trait values that maximise fitness as defined by Eq. (2.5); red circles show the mean population trait values after 100 generations of the GA. For each new noise level $s$, the GA was initialised with the trait values at the end of the previous simulation. Noise level was first increased in logarithmic increments from $10^{-6}$ to 1 (labelled 'increasing $s^{\prime}$ ) and then decreased in logarithmic decrements back down to $10^{-6}$ (labelled 'decreasing $s$ '). Black dotted line in (b) shows the deterministically optimum speed $v=v_{\text {det }}$. Population size $N=100$; mutation size constants $\sigma_{n}=0.1, \sigma_{v}=0.1 v_{\text {det }}$; maximum fecundity $n_{\max }=10^{6}$.

reduced, the population remains trapped in the high-fecundity local optimum, but the swimming speed evolves back down towards the deterministic optimum. In other words, the hysteresis observed in the population fecundity (Fig. 3a) is not present in the population swimming speed (Fig. 3b).

\section{Discussion}

The trade-off between producing many small eggs or fewer large ones has been investigated empirically (Duarte and Alcaraz, 1989; Elgar, 1990) and theoretically (Winemiller and Rose, 1993; Andersen et al., 2008). Stochasticity in the growth trajectories of fish larvae is clearly a major factor in the high-fecundity life histories of many marine fish species (Pitchford et al., 2005). In this paper, we have explored the interplay between fecundity and another heritable trait, larval swimming speed. We have shown that, in low-noise environments, the evolutionarily stable strategy is to swim at the speed that maximises the mean net growth rate, which is a balance between the prey encounter rate and the metabolic cost. This is the result of a simple deterministic optimisation. In high-noise environments, it becomes advantageous to swim faster than the deterministic optimum. This reduces the mean net growth rate, but increases the variance, and thereby increases the likelihood of having at least one offspring reach reproductive maturity. The evolutionarily stable swimming speed was estimated in two ways, which gave the same results: (i) by minimising the expected time taken for at least one offspring to reach maturity; (ii) by simulating the evolution of a population using a genetic algorithm. 
Considering the huge difference in size between the larvae and adults of most marine fish species, there is remarkably little variation in egg mass, with many species producing eggs close to $1 \mathrm{mg}$ (Ware and Lambert, 1985; Rijnsdorp and Ibelings, 1989; Chambers and Waiwood, 1996; Mendiola et al., 2006). This suggests that there may be physiological constraints that prevent production of viable eggs much smaller than this. For example, hydrodynamic factors severely limit the ability of small larvae to obtain an adequate food supply (China and Holzman, 2014). There is a strong correlation between an individual's reproductive effort (total mass of eggs produced) and its fecundity (Duarte and Alcaraz, 1989), suggesting that, given an increase in biomass available for reproduction, adult fish produce more eggs rather than larger eggs. These empirical observations are consistent with our model assumption that there is a maximum number of offspring that can be produced in a single spawning bout, corresponding to a minimum egg size.

The FishBase database (www.fishbase.org) is the principal repository for fish data, while the most comprehensive experimental study of larval swimming speed is Fisher et al. (2005). Of the 62 identified species studied by Fisher et al. (2005), FishBase provides fecundity estimates for four species: Lutjanus carponotatus (speed $52 \mathrm{~cm} / \mathrm{s}$, fecundity 7,074-748,959); Oxymonacanthus longirostris (speed $31.1 \mathrm{~cm} / \mathrm{s}$, fecundity 200-300); Dascyllus aruanus (speed 24 $\mathrm{cm} / \mathrm{s}$, fecundity 1,500-2,000); Plectropomus leopardus (speed $31.5 \mathrm{~cm} / \mathrm{s}$, fecundity 457,900$)$ (fecundity data extracted using rfishbase). The two species with the fastest swimming larvae (L. carponotatus and P. leopardus) have fecundities 2-3 orders of magnitude larger than the other two species.

This is consistent with our theoretical predictions for this long-standing evolutionary problem, but cannot be regarded as corroboration due to the small sample size. The quoted speeds are for settlement-stage juvenile fish and are based on measurements of maximum sustainable swimming speed; this is used as a proxy for foraging speed, but may mask other factors (Fisher and Leis, 2010). Moreover, empirical observations may reveal other differences in swimming and behaviour, involving for example diurnal changes in activity (Fisher and Bellwood, 2003). It is plausible that variations in swimming speed and movement behaviour will result in increasing variance in the context of the model developed here, and may thereby convey fitness benefits in a turbulent environment. Fish larvae also exhibit other traits: for example, some species possess oil globules which act as initial energy reserves (Fisher et al., 2007) and vary with body size and reproductive strategy. These are presumably subject to selection. The model presented here could be extended to account for this trait, possibly using simple descriptions of seasonality and unpredictability in the underlying food supply (James et al., 2003; Burrow et al., 2011).

We have presented results for a specific model of larval growth and mortality in Eq. (2.1). This simple model assumes a constant average growth rate $(r)$ and ignores density-dependent effects, such as competition for food or habitat, group defence against predators and cannibalism. In reality, interactions among individuals may be a function of relative body size, which could affect trade-offs involving fecundity. The model assumes there is no parental care: 
this is reasonable for most pelagic marine species, although there are some species that invest substantial effort into care of offspring (Duarte and Alcaraz, 1989). The model also ignores the substantial changes in the abundance and size of prey as an individual fish grows to maturity (Benoît and Rochet, 2004). An advantage of this simple model is that it only has a small number of parameters: the mean food encounter rate for stationary larvae, the proportion of parental mass used for reproduction, and the level of noise in the food encounter rate. The first two of these parameters were found not to have a major impact on evolutionarily stable outcomes. The noise level was shown to be a product of the patchiness of the prey distribution and the ratio of the mass of a prey item to the maturity mass. The reason for the latter effect is that, when fewer prey encounters are required to reach maturity, there is a higher probability of an individual offspring reaching maturity much faster than average. Patchy prey distributions have been argued to promote high-risk, high-reward strategies (Pitchford and Brindley, 2001).

The evolutionary stability of a high-fecundity strategy is robust to changes in model assumptions. For example, if both the mean and variance in the growth rate are proportional to size, rather than simply constant, Eq. (2.1) describes the logarithm of size and, for a given initial mass, results in the same hitting-time distribution. The trade-off between fecundity and initial mass results in slightly different fitness landscapes (Fig. 1), but the switch from low fecundity, slow swimming to high fecundity, fast swimming with increasing noise still occurs. When a resident population is following a high-fecundity strategy, egg size is by definition very small. A mutant with the same total reproductive effort but higher fecundity experiences little disadvantage from the further decrease in egg size, but benefits from having a greater number of tickets in the lottery of larval growth and survival. This effect is clearly at work in the genetic algorithm results in Fig. 2e, h, which show a steady evolution in the direction of increasing fecundity, consistent with empirical observation (Duarte and Alcaraz, 1989). Physiological and hydrodynamic factors are likely to put a lower limit on viable egg size (Levitan, 1993; China and Holzman, 2014), and we hypothesise that producing eggs around this size will be evolutionarily stable in many cases. An exception to this would be if there is sensitive dependence of larval survival on egg size, even at very small egg sizes. This could occur if the hatchlings' mass-specific growth rate is low relative to their mortality rate (Houde, 1997; Law et al., 2014).

In the model, we assumed that fish reproduce only once on reaching maturity and then die. In reality, mature fish of large species have a relatively low mortality rate and can survive for several years and undergo multiple reproductive bouts. The genetic algorithm could be generalised to include this by allowing non-overlapping generations. This would increase the advantage of having offspring reach maturity at an early age because this will allow multiple spawning bouts and lead to a faster increase in representation in the gene pool. This may enhance the selection pressure for high-risk, high-reward strategies like the one identified in this model, because there would be even greater benefit to having offspring in the extreme tail of the growth rate distribution. 
We chose to study larval swimming speed because it is a variable trait that is a key determinant of prey encounter rate. But our results illustrate a wider point: when a particular trait has a combination of deterministic and stochastic effects, its optimal value will depend on the level of stochasticity (Currey et al., 2007). Optimising mean values, like the mean growth rate, is not likely lead to an evolutionarily stable strategy when stochastic effects are strong and when only a small fraction of offspring reach reproductive maturity.

\section{Appendix}

We define dimensionless variables

$$
\hat{X}=\frac{X}{x_{m}}, \quad \hat{t}=\frac{t}{t_{\text {ref }}}, \quad \hat{v}=\frac{v}{v_{\text {det }}},
$$

where $t_{\text {ref }}=4 c x_{m} /\left(b^{2} x_{p}^{2}\right)$ and $v_{\operatorname{det}}=b x_{p} /(2 c)$, which is the swimming speed that maximises the expected growth rate, i.e. the deterministic optimum. Then Eq. (2.1) becomes

$$
d \hat{X}=\hat{r} d \hat{t}+\hat{\sigma} d \hat{W}
$$

where

$$
\begin{aligned}
\hat{r} & =\frac{4 a c}{b^{2} x_{p}}+2 \hat{v}-\hat{v}^{2}, \\
\hat{\sigma}^{2} & =S \frac{x_{p}}{x_{m}}\left(\frac{4 a c}{b^{2} x_{p}}+2 \hat{v}\right)
\end{aligned}
$$

and $\hat{W}=W / t_{\text {ref }}^{1 / 2}$ is a standard Brownian motion with respect to dimensionless time $\hat{t}$. The initial condition in the new variables is $\hat{X}(0)=x_{0} / x_{m}$ and the fish reaches maturity when $\hat{X}=1$. Dropping the hats, this is equivalent to Eq. (2.1) with the growth rate and diffusivity given in Eqs. (2.2) and (2.3).

\section{References}

Andersen, K. H., Beyer, J., Pedersen, M., Andersen, N. G., and Gislason, H. (2008). Lifehistory constraints on the success of the many small eggs reproductive strategy. Theoretical Population Biology, 73(4):490-497.

Benoît, E. and Rochet, M.-J. (2004). A continuous model of biomass size spectra governed by predation and the effects of fishing on them. Journal of Theoretical Biology, 226(1):9-21.

Burrow, J. F., Horwood, J. W., and Pitchford, J. W. (2011). The importance of variable timing and abundance of prey for fish larval recruitment. Journal of Plankton Research, page fbr015.

Chambers, R. C. and Leggett, W. C. (1996). Maternal influences on variation in egg sizes in temperate marine fishes. American Zoologist, 36(2):180-196.

Chambers, R. C. and Waiwood, K. G. (1996). Maternal and seasonal differences in egg sizes and spawning characteristics of captive Atlantic cod, Gadus morhua. Canadian Journal of Fisheries and Aquatic Sciences, 53(9):1986-2003.

Chick, J. H. and Van Den Avyle, M. J. (2000). Effects of feeding ration on larval swimming speed and responsiveness to predator attacks: implications for cohort survival. Canadian Journal of Fisheries and Aquatic Sciences, 57(1):106-115.

China, V. and Holzman, R. (2014). Hydrodynamic starvation in first-feeding larval fishes. Proceedings of the National Academy of Sciences, 111(22):8083-8088. 
Currey, J. D., Pitchford, J. W., and Baxter, P. D. (2007). Variability of the mechanical properties of bone, and its evolutionary consequences. Journal of The Royal Society Interface, 4(12):127-135.

Darowski, K., Takashima, F., and Law, Y. (1988). Bioenergetic model of planktivorous fish feeding, growth and metabolism: theoretical optimum swimming speed of fish larvae. Journal of Fish Biology, 32(3):443-458.

Duarte, C. M. and Alcaraz, M. (1989). To produce many small or few large eggs: a sizeindependent reproductive tactic of fish. Oecologia, 80(3):401-404.

Elgar, M. A. (1990). Evolutionary compromise between a few large and many small eggs: comparative evidence in teleost fish. Oikos, 59:283-287.

Fisher, R. and Bellwood, D. R. (2003). Undisturbed swimming behaviour and nocturnal activity of coral reef fish larvae. Marine Ecology Progress Series, 263:177-188.

Fisher, R. and Leis, J. M. (2010). Swimming speeds in larval fishes: from escaping predators to the potential for long distance migration. In Fish locomotions: An eco-ethological perspective, chapter 11, pages 333-373. Science Publishers.

Fisher, R., Leis, J. M., Clark, D. L., and Wilson, S. K. (2005). Critical swimming speeds of late-stage coral reef fish larvae: variation within species, among species and between locations. Marine Biology, 147(5):1201-1212.

Fisher, R., Sogard, S. M., and Berkeley, S. A. (2007). Trade-offs between size and energy reserves reflect alternative strategies for optimizing larval survival potential in rockfish. Marine Ecology Progress Series, 344:257-270.

Grimmett, G. and Stirzaker, D. (1992). Probability and random processes. Oxford Univ Press.

Houde, E. D. (1997). Patterns and consequences of selective processes in teleost early life histories. In Early life history and recruitment in fish populations, chapter 6, pages 173-196. Springer.

James, A., Pitchford, J. W., and Brindley, J. (2003). The relationship between plankton blooms, the hatching of fish larvae, and recruitment. Ecological Modelling, 160(1):77-90.

Jennings, S. and Warr, K. J. (2003). Environmental correlates of large-scale spatial variation in the $\delta 15 \mathrm{~N}$ of marine animals. Marine Biology, 142(6):1131-1140.

Law, R., Plank, M. J., and Kolding, J. (2014). Balanced exploitation and coexistence of interacting, size-structured, fish species. Fish and Fisheries.

Levitan, D. R. (1993). The importance of sperm limitation to the evolution of egg size in marine invertebrates. American Naturalist, 141:517-536.

MacKenzie, B. R. and Kiørboe, T. (1995). Encounter rates and swimming behavior of pausetravel and cruise larval fish predators in calm and turbulent laboratory environments. Limnology and Oceanography, 40(7):1278-1289.

Maynard-Smith, J. (1982). Evolution and the theory of games. Cambridge University Press.

Mendiola, D., Alvarez, P., Cotano, U., Etxebeste, E., and de Murguia, A. M. (2006). Effects of temperature on development and mortality of atlantic mackerel fish eggs. Fisheries Research, 80(2):158-168.

Pitchford, J. W. and Brindley, J. (2001). Prey patchiness, predator survival and fish recruitment. Bulletin of Mathematical Biology, 63(3):527-546.

Pitchford, J. W., James, A., and Brindley, J. (2003). Optimal foraging in patchy turbulent environments. Marine Ecology Progress Series, 256:99-110.

Pitchford, J. W., James, A., and Brindley, J. (2005). Quantifying the effects of individual and environmental variability in fish recruitment. Fisheries Oceanography, 14(2):156-160.

Rijnsdorp, A. D. and Ibelings, B. (1989). Sexual dimorphism in the energetics of reproduction and growth of North Sea plaice, Pleuronectes platessa L. Journal of Fish Biology, $35(3): 401-415$.

Tsuda, A., Sugisaki, H., Ishimaru, T., Saino, T., and Sato, T. (1993). White-noise-like distribution of the oceanic copepod Neocalanus cristatus in the subarctic North Pacific. Mar. Ecol. Prog. Ser, 97:39-46.

Ware, D. M. and Lambert, T. C. (1985). Early life history of atlantic mackerel (Scomber scombrus) in the southern Gulf of St. Lawrence. Canadian Journal of Fisheries and Aquatic Sciences, 42(3):577-592.

Winemiller, K. O. and Rose, K. A. (1993). Why do most fish produce so many tiny offspring? American Naturalist, 142:585-603. 